\title{
The Finite Embeddability Property for some Noncommutative Knotted Extensions of FL
}

\author{
Riquelmi Cardona ${ }^{1}$ \\ University of Denver, Denver, Colorado, U.S.A. \\ rcardon3@du.edu
}

\section{Introduction.}

A (non-trivial) knotted rule is an inequality of the form $x^{m} \leq x^{n}$ for $m \neq n, m \geq 1, n \geq 0$. Knotted rules were introduced in 2 .

Consider the variety $\mathcal{V}$ of residuated lattices that satisfy $x y x=x^{2} y$ and any knotted rule $x^{m} \leq x^{n}$. We will show that $\mathcal{V}$ has the finite embeddability property (FEP). Previously Van Alten proved in 4 that the FEP holds for commutative residuated lattices that satisfy any knotted rule. A class of algebras $\mathcal{K}$ is said to have the FEP, if for every algebra $\mathbf{A}$ in $\mathcal{K}$ and every finite partial subalgebra $\mathbf{B}$ of $\mathbf{A}$, there exists a finite algebra $\mathbf{D}$ in $\mathcal{K}$ such that $\mathbf{B}$ embeds into $\mathbf{D}$. $\mathbf{B}$ is a finite partial subalgebra of $\mathbf{A}$, if $B$ is a finite subset of $A$ and each $n$-ary operation $f^{\mathbf{A}}$ on $A$ induces a partial operation $f^{\mathbf{B}}$ on $B$ defined as:

$$
f^{\mathbf{B}}\left(b_{1}, \ldots, b_{n}\right)= \begin{cases}f^{\mathbf{A}}\left(b_{1}, \ldots, b_{n}\right), & \text { if } f^{\mathbf{A}}\left(b_{1}, \ldots, b_{n}\right) \in \mathbf{B} . \\ \text { undefined, } & \text { if } f^{\mathbf{A}}\left(b_{1}, \ldots, b_{n}\right) \notin \mathbf{B} .\end{cases}
$$

We have that if $\mathcal{V}$ possesses the FEP, then $\mathcal{V}$ has the finite model property as well. Namely, it is generated by its finite members.

A residuated frame is a structure of the form $\mathbf{W}=\left(W, W^{\prime}, N, \circ, \|, / /\right)$, where $(W, \circ)$ is a monoid and $N \subseteq W \times W^{\prime}$ is a nuclear relation on $(W, \circ)$ with respect to $\mathbb{\|}, / /$. The Galois algebra of $\mathbf{W}$ is denoted by $\mathbf{W}^{+}$. We define $\mathbf{W}^{+}=\left(\gamma_{N}[\wp(W)], \cap, \cup_{\gamma_{N}}, \circ_{\gamma_{N}}, \backslash, /\right)$. We have that the latter is a residuated lattice. See 11 for details.

Consider a $\mathbf{A} \in \mathcal{V}$ and $\mathbf{B}$ a finite partial subalgebra of $\mathbf{A}$ such that $B=\left\{b_{1}, b_{2}, \ldots, b_{k}\right\}$. Define $(W, \circ, 1)$ to be the submonoid of $\mathbf{A}$ generated by $B$. By associativity, a unary linear polynomial of $(W, \circ, 1)$ is of the form $u(x)=y \circ x \circ w$ for $y, w \in W$. Such polynomials are also known as sections and we denote the set of all sections by $S_{W}$. Let $W^{\prime}=S_{W} \times B$, and define $x N(u, b)$ by $u^{\mathbf{A}}(x) \leq^{\mathbf{A}} b$. During the rest of the paper we will use $u(x)$ to represent $u^{\mathbf{A}}(x)$ depending from the context. Given $y \in W$ and $u \in S_{W}$, define sections $u^{\prime}(x)=u(x \circ y)$ and $u^{\prime \prime}(x)=u(y \circ x)$. We will also use the notation $u^{\prime}=u\left({ }_{-} \circ x\right)$ and $u^{\prime \prime}=u\left(x \circ{ }_{-}\right)$. Define $y \|(u, b)=\left\{\left(u\left(y \circ{ }_{-}\right), b\right)\right\}$ and $(u, b) \| y=\left\{\left(u\left(\__{-} \circ y\right), b\right)\right\}$. Then $\mathbf{W}_{\mathbf{A}, \mathbf{B}}=\left(W, W^{\prime}, N, \circ, \|, / /\right)$ is a residuated frame. In the construction from [1], it is shown that the map $b \mapsto\{(i d, b)\} \triangleleft$ is an embedding of the partial subalgebra $\mathbf{B}$ of $\mathbf{A}$ into $\mathbf{W}_{\mathbf{A}, \mathbf{B}}^{+}$. For the construction we will use the results that $\mathbf{W}_{\mathbf{A}, \mathbf{B}}^{+}$and $\mathbf{A}$ belong $\mathcal{V}$ and that the closed sets $\{(u, b)\}^{\triangleleft}$ for $u \in S_{W}, b \in B$ form a basis for $\mathbf{W}_{\mathbf{A}, \mathbf{B}}^{+}$.

\section{The construction.}

We divide the problem in two cases. First we consider the pomonoid class $\mathcal{P}$ that satisfies $x y x=x^{2} y$ and $x^{m} \leq x^{n}$, for $m>n$. To show that $\mathcal{V}$ has the FEP, we will find a pomonoid 
$\mathbf{F} \in \mathcal{V}$ such that $\left(W, \circ, 1, \leq^{\mathbf{A}}\right)$ is a homomorphic image of $\mathbf{F}$. We will utilize the structure of $\mathbf{F}$ to prove that the basis of closed sets of $\mathbf{W}_{\mathbf{A}, \mathbf{B}}^{+}$is finite. One candidate for $\mathbf{F}$ is the free algebra over $\mathcal{P}$.

\subsection{The free algebra in $k$ generators over $\mathcal{P}$.}

First, we identify and construct the free algebra $\mathbf{F}_{\mathbf{M}}$ over $k$ generators of the variety $\mathcal{M}$ of monoids such that $x y x=x^{2} y . \mathbf{F}_{\mathbf{M}}$ will be the monoid reduct of the $\mathbf{F}$. The defining equation of this variety implies that $x^{k} y x^{\ell}=x^{k+\ell} y$. Hence, expressions in these monoids can be written using every generator at most once. In this case every element can be identified by the order in which the generators appear and their exponents.

For instance, if we have 5 generators $\left\{z_{1}, z_{2}, z_{3}, z_{4}, z_{5}\right\}$, then $z_{5}^{3} z_{1}^{4} z_{3}^{2}$ can be encoded by the exponents of the generators $(4,0,2,0,3)$ and the order in which they appeared 513 . In the order 513 we only include the indices of the generators that have exponents greater than 0 . With this encoding, $z_{5}^{3} z_{1}^{4} z_{3}^{2}$ would be represented as $((4,0,2,0,3), 513)$.

When we multiply two elements, the exponents of the corresponding generators are added. On the other hand, the order of the generators is consolidated. If we consider the generators without the exponents, the defining equation $x y x=x^{2} y$ becomes $x y x=x y$. The last equation captures the behavior of the order of the generators.

The order of the generators or signature of the expressions will be modeled by a monoid that satisfies $x y x=x y$. Let's consider the free idempotent monoid in $k$ generators $\{1,2 \ldots, k\}$ that satisfies the equation $\forall x, y(x y x=x y)$. In this monoid $\mathbf{S}=(S, \cdot \varepsilon), S$ consists of all the words where each generator appears at most once. The operation is concatenation and when a generator appears twice or more we preserve only the leftmost one. We have that $S$ is a finite set.

For $s \in S$, let $|s|$ denote the length of $s$. When $i>0$, define $s_{i}$ to represent the $i$ th position in $s$, where $i \leq|s|$. Define $\bar{s}=\left\{s_{i}: i \leq|s|\right\}$, the set of elements that appear in $s$.

Now, the operation in $\mathbf{F}_{\mathbf{M}}$ will be defined by addition in $\mathbb{N}^{k}$ for the first coordinate and multiplication in $\mathbf{S}$ for the second coordinate.

We have enough information to determine the free monoid of $\mathcal{M}$. Notice that $\left(\mathbb{N}^{k},+, 0\right), \mathbf{S} \in$ $\mathcal{M}$. Let $F=\left\{(\vec{x}, s) \in \mathbb{N}^{k} \times S: \bar{s}=\operatorname{supp}(\vec{x})\right\} . \quad F$ is closed under multiplication, hence $\mathbf{F}_{\mathbf{M}}=\left(F,{ }^{\mathbf{F}}, 0\right)$ is a submonoid of $\mathbb{N}^{k} \times \mathbf{S}$. Clearly $\mathbf{F}_{\mathbf{M}}$ is in $\mathcal{M}$. We will write $x \in F$ as $x=\left(\vec{x}, s_{x}\right)$.

Lemma 2.1. $\mathbf{F}_{\mathbf{M}}=(F, \cdot \mathbf{F}, 0)$ is the free algebra in $\mathcal{M}$ on $k$ generators. Moreover, the set $F$ is generated by the vectors: $z_{1}=((1,0, \ldots, 0), 1), z_{2}=((0,1, \ldots, 0), 2), \ldots, z_{k}=((0,0, \ldots, 1), k)$. Also, the identity vector is $0=(\overrightarrow{0}, \varepsilon)$.

For $m>n$ let's define a relation $\leq_{n}^{m}$ on the non negative integers $\mathbb{N}$ by $u \leq_{n}^{m} v$ if and only if $u=v$, or $n \leq v<u$ and $u \equiv v(\bmod m-n)$. This relation is a partial order on $\mathbb{N}$ that is compatible with + .

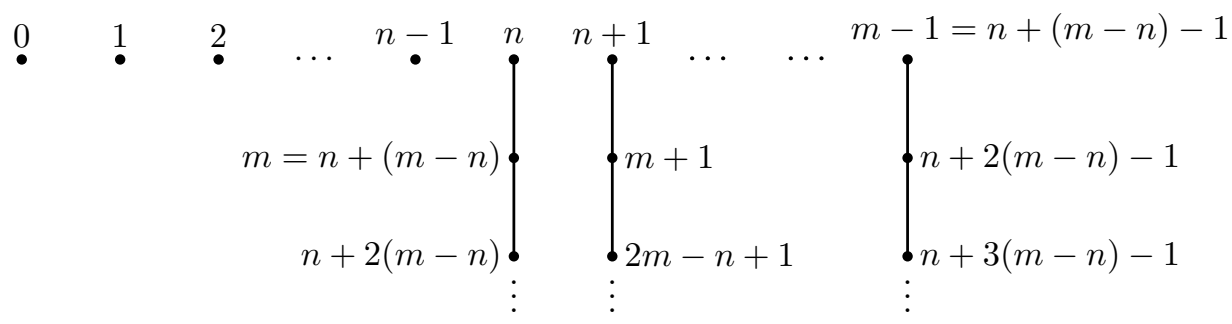


A partially ordered set is said to be well partially ordered if it has no infinite antichains and no infinite descending chains. For instance, $\mathbb{N}$ with the usual order, namely $\langle\mathbb{N}, \leq\rangle$, is well partially ordered. If $\langle P, \leq\rangle$ is well partially ordered, then it is known that for each $k \in \mathbb{N}, P^{k}$ is well partially ordered under the direct product ordering of $P^{k}$. Furthermore, disjoint unions, subposets and homomorphic images of well partially ordered sets are well partially ordered.

Notice that $\left\langle\mathbb{N}, \leq_{n}^{m}\right\rangle$ is the disjoint union of $n$ one-element chains and $(m-n)$ chains isomorphic to $\left\langle\mathbb{Z}^{-}, \leq\right\rangle$. Thus, it is dually well partially ordered. This order can be extended to the direct product $\left\langle\mathbb{N}^{k}, \leq_{n}^{m}\right\rangle$, which is dually well partially ordered as well.

We have that $\left\langle\mathbb{N}^{k}, \leq_{n}^{m}\right\rangle$ and $\langle S,=\rangle$ are dually well partially ordered sets. Consider $\mathbf{F}$ under the order $\leq^{F}$, defined as $\forall x, y \in F,\left(x \leq^{F} y\right)$ iff $\vec{x} \leq_{n}^{m} \vec{y}$ and $s_{x}=s_{y}$. Then $\left(F,{ }^{\mathbf{F}}, 0, \leq^{F}\right)$ is a subpomonoid of the direct product $\left(\mathbb{N}^{k},+, 0, \leq_{n}^{m}\right) \times(S, \cdot, \varepsilon,=)$. Hence $\left\langle F, \leq^{F}\right\rangle$ is a dually well partially ordered set. Furthermore, $\leq^{F}$ is compatible with. $\mathbf{F}$ because $\leq_{n}^{m}$ and $=$ are compatible with the corresponding operations.

Lemma 2.2. $\left(F,{ }^{\mathbf{F}}, 0, \leq^{F}\right)$ is the free object in $\mathcal{P}$.

\subsection{Case $m>n$.}

Recall that $B=\left\{b_{1}, \ldots, b_{k}\right\}$ and $Z$ is the set of generators of $\mathbf{F}$. Let $h_{1}: Z \rightarrow W$ be the map that sends $z_{i} \mapsto b_{i}$ for each $i=1, \ldots, k$ and extend it to a pomonoid homomorphism $h:\left(F,{ }^{\mathbf{F}}, 0, \leq^{F}\right) \rightarrow\left(W, \circ, 1, \leq^{\mathbf{A}}\right)$ by the universal mapping property. The map $h$ is surjective because $B$ generates $(W, \circ, 1)$.

Consider the new frame $\mathbf{W}_{\mathbf{A}, \mathbf{B}}^{\mathbf{F}}=\left(F, W^{\prime}, h \circ N, \cdot{ }^{\mathbf{F}}, / / h, \|_{h},\{1\}\right)$, where $x(h \circ N) z$ iff $h(x) N z$, and $x \|_{h} z=h(x) \Downarrow z$ and $z / / h y=z / / h(y)$. Then $h \circ N$ is nuclear, so $\mathbf{W}_{\mathbf{A}, \mathbf{B}}^{\mathbf{F}}$ is a residuated frame. To prove the finite embeddability property we will show that $\mathbf{W}_{\mathbf{A} . \mathbf{B}}^{+}$is finite. It suffices to prove that it possesses a finite basis of sets $\{z\}^{\triangleleft_{N}}=\{x \in W: x N z\}$, for $z \in W^{\prime}$. As $h$ is surjective, it is enough to show that there are finitely many sets of the form $\{z\}^{\triangleleft}=\{x \in F: x(h \circ N) z\}$, for $z \in W^{\prime}$.

For $x \in F$, and $(u, b) \in W^{\prime}$, we have $x \in\{(u, b)\}^{\triangleleft}$ iff $u(h(x)) \leq^{\mathbf{A}} b$ iff $h(v(x)) \leq^{\mathbf{A}} b$, for some $v \in S_{F}$ such that $h(v)=u$, since $h$ is a surjective homomorphism. Equivalently, $v(x) \in h^{-1}\left[\downarrow_{A} b\right]$, for some $v \in h^{-1}(u)$. Now, $h^{-1}\left[\downarrow_{A} b\right]$ is a downset in $F$. Since $F$ is dually well partially order, we have that $h^{-1}\left[\downarrow_{A} b\right]=\downarrow D_{b}$, for some finite $D_{b} \subseteq F$.

Hence $v(x) \leq{ }^{F} d$ for some $d \in D_{b}$. Define $\frac{d}{v}=\{x \in F: v(x) \leq F d\}$, where $v=y \cdot{ }^{\mathbf{F}}{ }_{-}{ }^{\mathbf{F}} w$ for some $y, w \in F$.

Lemma 2.3. For a fixed $d \in D_{b}$, there exists finitely many choices for the sets $\frac{d}{v}$.

Given that

$$
\{(u, b)\}^{\triangleleft}=\bigcup_{d \in D_{b}} \frac{d}{v},
$$

and $D_{b}$ is finite, we conclude that for a fixed $b$, there exists finitely many $\{(u, b)\}^{\triangleleft}$. This and the fact that $B$ is a finite set concludes the proof.

\subsection{Case $m<n$.}

Define a relation $\leq_{m}^{n}$ (notice that the bigger value is the subindex in this order) on the $\mathbb{N}$ by $u \leq_{n}^{m} v$ if and only if $u=v$, or $m \leq u<v$ and $v \equiv u(\bmod m-n)$. This order is the dual of the order $\leq_{m}^{n}$, i.e., $\leq_{n}^{m}=\geq_{m}^{n}$. This implies that $\left\langle\mathbb{N}^{k}, \leq_{n}^{m}\right\rangle$ is well partially ordered.

As before, we construct the free pomonoid $\left(F, \cdot{ }^{\mathbf{F}}, 0, \leq^{F}\right)$ for this class. By the universal mapping property, there exists a surjective homomorphism $h:\left(F,{ }^{\mathbf{F}}, 0\right) \rightarrow(W, \circ, 1)$. Following 
the previous construction we conclude that it is sufficient to show that there are only finitely many closed sets of the form $\{(u, b)\}^{\triangleleft}$ with $u \in S_{W}, b \in B$.

We use a characterization of well partially ordered sets taken from Nash-Williams 3 . Let $\langle P, \leq\rangle$ be a poset. An infinite sequence $p_{1}, p_{2}, \ldots$ of elements of $P$ is called bad when $i<j$ implies that $p_{i} \leq \leq p_{j}$. Note that an infinitely descending chain or antichain would be a bad sequence. A poset is well partially ordered if and only if it has no bad sequences.

Now, for each $b \in B$, define $C_{b}=\left\{\{(u, b)\}^{\triangleleft}: u \in S_{W}\right\}$. The result follows from the next two lemmas. We omit the proofs here.

Lemma 2.4. For each $b \in B,\left\langle C_{b}, \supseteq\right\rangle$ is well partially ordered.

By the above lemma $\left\langle C_{b}, \supseteq\right\rangle$ has no infinite antichains or descending chains.

Lemma 2.5. $\left\langle C_{b}, \supseteq\right\rangle$ has no infinite ascending chains.

Since $\left\langle C_{b}, \supseteq\right\rangle$ has no infinite ascending chains, infinite descending chains and no infinite antichains, $C_{b}$ is finite for every $b \in B$. Thus, there are finitely many sets of the form $\{(u, b)\}^{\triangleleft}$ because $B$ is finite.

Theorem 2.6. The variety $\mathcal{V}$ has the finite embeddability property and the finite model property.

\section{Generalization}

The previous proof can be tailored to prove the FEP for the variety $\mathcal{V}(r)$ of residuated lattices such that for some $a_{i} \in \mathbb{N}$

$$
x y_{1} x y_{2} x \cdots x y_{r} x=x^{a_{0}} y_{1} x^{a_{1}} y_{2} x^{a_{2}} \cdots x^{a_{r-1}} y_{r} x^{a_{r}} \text {, where } \sum_{i=0}^{r} a_{i}=r+1 \text { and } \prod_{i=0}^{r} a_{i}=0,
$$

and $x^{m} \leq x^{n}$ for $m<n, m \geq 1$. In this case, we will prove the FEP for a variety containing $\mathcal{V}(r)$. For the previous construction it was important to prove that our signature monoid $\mathbf{S}$ was finite. We will create a signature monoid that is finite and captures the behavior of our variety.

Lemma 3.1. The equation $x y_{1} x y_{2} x \cdots x y_{r} x=x^{a_{0}} y_{1} x^{a_{1}} y_{2} x^{a_{2}} \cdots x^{a_{r-1}} y_{r} x^{a_{r}}$ implies that for all $\ell \geq 2 r$, there exist natural numbers $p, q$, and $t$ such that

$$
x y_{1} x y_{2} x \cdots x y_{\ell} x=x y_{1} x y_{2} x \cdots x y_{p-1} x^{t} y_{p} y_{p+1} \cdots y_{\ell-q+1} x y_{\ell-q+2} x \cdots y_{\ell} x,
$$

where $t=\ell+2-(p+q) \geq 0$.

Then our signature set will be bounded because every variable will appear at most in $q$ places.

Claim. The variety $\mathcal{V}(r)$ for all $r \in \mathbb{Z}^{+}$has the FEP.

\section{References}

[1] N. Galatos and P. Jipsen, Residuated frames with applications to decidability, Transactions of the American Mathematical Society 365 (2013), no. 3, 1219-1249.

[2] R Hori, H. Ono, and H. Schellinx, Extending intuitionistic linear logic with knotted structural rules., Notre Dame Journal of Formal Logic 35 (1994), no. 2, 219-242.

[3] C. Nash-Williams, On well-quasi-ordering infinite trees, Mathematical proceedings of the cambridge philosophical society, 1965 , pp. 697-720.

[4] C. Van Alten, The finite model property for knotted extensions of propositional linear logic, Journal of Symbolic Logic 70 (2005), no. 1, 84-98. 